\title{
Automated control of anesthesia ten years later: futuristic novelty or present day reality
}

\author{
Peter S. A. Glass, MD
}

Published online: 28 May 2010

(C) Canadian Anesthesiologists' Society 2010

The specialty of anesthesia evolved following the discovery that drugs were able to create a reversible state of unconsciousness enabling surgical interventions to be performed. The need for human control over such powerful and potentially dangerous compounds was implicit in developing the specialty. The notion of anesthesia as an art practiced by professionals versed in human physiology and pharmacology has not been challenged until recently.

In essence, we have known very little about the fundamental nature of anesthesia, and, without a doubt, we have not had means to measure it. John Snow, one of the earliest and most pre-eminent anesthesiologist of his time, stated early on in his career that the greatest challenge is knowing when the ether had been carried far enough, ${ }^{1}$ i.e., that the practice of anesthesia was an art rather than a precise science. What has changed to make us believe that we can hand over the control of the anesthetic state to a robot or, more precisely, to a computer-controlled algorithm? The manuscript by Hemmerling et al. in this issue of the Journal certainly forces us to consider whether relinquishing control would be a viable option for providing anesthesia. $^{2}$

Our understanding of the nature of anesthesia and our ability to measure at least a component of the anesthetic state have evolved over the past 15 years. Seminal work by Rampil $^{3,4}$ and Antognini ${ }^{5}$ has contributed enormously to our knowledge of the anesthesia paradigm. Rampil demonstrated that the ablation of a movement response to a noxious stimulus is due to the action of a volatile anesthetic on a spinal process rather than on a process of the brain.

P. S. A. Glass, MD ( $\square)$

Department of Anesthesiology, Stony Brook University Medical Center, HSC Level 4-060, Stony Brook, NY 11794-8480, USA

e-mail:pglass@notes.cc.sunysb.edu
Antognini demonstrated that a significantly higher minimum alveolar concentration was required to prevent movement when anesthetizing a goat's brain independently of the spinal cord than was required when exposing both the brain and the spinal cord to the volatile anesthetic. These two pieces of information clearly indicated that a critical suppression of the spinal cord is required to achieve anesthesia, yet the spinal cord is not responsible for loss of consciousness, an essential element of general anesthesia. Soon after this work, it was shown that opiates could reduce the minimum alveolar concentration (MAC) of a volatile anesthetic to a value equal to their MAC awake concentration. That is to say, as sufficient opiate is added during the administration of a volatile anesthetic to provide general anesthesia, the concentration of volatile anesthetic is reduced to that which is just needed to render an individual unresponsive to a verbal stimulus. This is not too dissimilar to using a combination of spinal/epidural analgesia and adding just enough volatile anesthetic to render the patient unconscious. Based on this information, I would propose that anesthesia involves initially rendering the patient unconscious by a mechanism within a consciousness centre in the brain and, secondly, preventing a noxious stimulus from reaching this consciousness centre. This knowledge also makes us appreciate that we must be able to measure the effects of the anesthetic on both brain function and spinal cord function if we are to create an automated system to control anesthesia- a challenge.

In the late $1940 \mathrm{~s}$ and early $1950 \mathrm{~s}$, Bickford began to evaluate the electroencephalogram (EEG) as a measure of anesthetic drug effect and its correlation to loss of consciousness. At this early stage, an automated system of drug delivery to provide anesthesia started to develop. ${ }^{6}$ The EEG is a very complex wave pattern that several investigators have tried to reduce to a single derived value 
(e.g., spectral edge, median EEG frequency). With these derived measures of the EEG, most anesthetics result in a biphasic pattern as they provide increasing sedation and loss of consciousness. The bispectral index (BIS), another derived index of the EEG, was developed specifically as a linear measure of increasing sedation and loss of consciousness (especially in the value range of 50-100). This development has provided a tool to measure at least one component of the anesthetic state, thereby enabling automated control of this component.

The engineering algorithms involved in creating a closed-loop system are well-known. Biological systems, however, tend to provide far greater challenges. A simple automated control system with which we are all familiar is the cruise control on our motor vehicles. The challenges of controlling a biological system include the time-varying changes in the relationship between drug dose and drug concentration and inter- and intra-individual variability in sensitivity (i.e., the changing relationship between drug concentration and effect). Thus, there is no simple relationship between input and output. A variety of solutions have been developed to resolve these problems, including the use of pharmacokinetic models to account for the time varying relationship between dose and effect-site concentration, adaptive controllers, or fuzzy logic to account for differences in sensitivity. ${ }^{7-11}$

Thus, engineering solutions have been developed for the automated control of a biological system, and we have at least one component of anesthesia we can place under automated control. Hemmerling et al., like several previous authors, demonstrate the use of an automated closed-loop system to administer propofol to maintain BIS within a narrower band. In fact, the system performed better than human (manual) control in maintaining the BIS at or close to its set point. The algorithm the authors used for their closed-loop system differs somewhat from that used by other investigators, but it follows general engineering principles. The authors also showed that emergence times were, on average, three minutes shorter in the patients administered propofol using the closed-loop system - thus implying that their closed-loop system performed better than human propofol administration in terms of both intraoperative control of hypnosis and outcomes. Does this imply that automated control provides better anesthesia than manual control and thus is a "present reality"? In a previous editorial almost ten years ago regarding a similar evaluation of automated closed-loop control of anesthesia, I also challenged the notion of whether demonstrating simply that automated closed-loop systems were able to control a BIS value precisely would translate into demonstrating that such systems are truly better than humans administering the drugs required to obtain and maintain anesthesia. $^{12}$
When assessing the performance of an automated closed-loop anesthesia device, the attributes the authors are demonstrating need to be evaluated critically in the context of the nature of anesthesia. What Hemmerling et al.'s study demonstrates is that the control algorithm used in this closed-loop system of propofol administration works well (or at least as well as human control) in maintaining a set/ target BIS value and, in so doing, provides for an adequate rate of recovery. As previously explained, anesthesia has at least two components, yet to date, all automated systems have controlled only the hypnotic portion. The interaction of noxious stimulation, analgesia, hypnosis, and anesthesia is complex. ${ }^{13,14}$ The relationship between analgesia and hypnosis in providing anesthesia is not linear, and noxious stimulation varies continuously throughout surgery. Controlling only the hypnotic component makes sense if analgesia is provided with a regional anesthetic so that only hypnosis is needed to complete a state of anesthesia. However, if opiates are provided for analgesia during times of intense noxious stimuli, a closed-loop system would respond by increasing the hypnotic to maintain the pre-set BIS value, whereas the more appropriate response may be to provide a brief period of increased analgesia. No closedloop system is able, as yet, to make such subtle decisions. In the study by Hemmerling et al., opiate dosing was at the discretion of the anesthesiologist, thus making the system only partially a closed-loop system for anesthesia (i.e., it was really a closed-loop hypnotic system rather than closed-loop anesthesia). To this end, it is interesting to observe that the authors chose a BIS value of 45 as the target, which is at the lower end of BIS values recommended to maintain during anesthesia. The patients were given a "hypnotic heavy" anesthetic, thereby minimizing the complex interaction between hypnosis analgesia and noxious stimulus. Thus, similar to all studies to date, Hemmerling et al.'s study does not demonstrate whether humans, when they are allowed to provide their best anesthetic management and are not constrained by a predetermined surrogate measure of only one component of the anesthetic, perform better or worse than the best closedloop control systems we presently have available. In addition, under the most challenging and varying conditions that present during anesthesia, can a closed-loop system provide better outcomes than a human administering the anesthetic?

These are far more difficult questions to answer. A good clinician will use the BIS and other signs of adequate/ inadequate anesthesia to guide the administration of both hypnotic and analgesic in an effort to provide an anesthetic in anticipation of the surgeon's delivery of noxious stimuli yet allowing for the rapid return of consciousness with a comfortable patient. Thus, I would propose that we have established that closed-loop hypnosis can be achieved very 
successfully. However, what needs to be shown is whether automated closed-loop administration of anesthetics is truly better than that achievable by an anesthesia provider. To accomplish this, we should not compare human ability to achieve a desired set point (i.e., BIS value) with that provided by a closed-loop system, but rather, we should compare the best anesthetic each anesthesia provider can administer. In addition, these systems should be tested under physiological extremes, e.g., hypotension/hypertension, etc. Only then can we determine if automated control of anesthesia is a futuristic novelty or a present day reality. I remain optimistic that closed-loop control of anesthesia ultimately will prove to be superior and will become routine in providing anesthesia.

\section{Surveillance automatisée de l'anesthésie, dix ans plus tard: innovation futuriste ou réalité actuelle?}

La spécialité qu'est l'anesthésie a vu le jour et évolué suite à la découverte que les médicaments pouvaient créer un état d'inconscience réversible, ce qui a permis la réalisation d'interventions chirurgicales. Le besoin d'une surveillance humaine de ces molécules si puissantes et potentiellement dangereuses faisait implicitement partie de l'évolution de la spécialité. La notion d'anesthésie en tant qu'art pratiqué par des professionnels maîtrisant la physiologie et la pharmacologie humaines n'a, jusqu'à récemment, jamais été remise en question.

Par essence, nos connaissances concernant la nature fondamentale de l'anesthésie sont très limitées et nous n'avons certainement pas eu les moyens de la mesurer. John Snow, l'un des premiers anesthésiologistes et l'un des plus éminents de son époque, déclarait, au début de sa carrière déjà, que le plus grand défi de l'anesthésiologiste est de savoir quand l'éther est allé suffisamment loin. ${ }^{1}$ En d'autres termes, la pratique de l'anesthésie est un art plutôt qu'une science exacte. Qu'est-ce qui a changé qui nous incite à croire que nous pouvons céder la surveillance de l'état anesthésique à un robot ou, plus précisément, à un algorithme informatique? Dans ce numéro du Journal, le manuscrit de Hemmerling et coll. nous pousse à réflexion: serait-ce une option viable que de céder la surveillance lors de la fourniture d'une anesthésie? ${ }^{2}$

$\mathrm{Au}$ cours des quinze dernières années, notre compréhension de la nature de l'anesthésie et notre capacité à mesurer au moins une composante de l'état anesthésique ont évolué. Les travaux précurseurs de Rampil ${ }^{3,4}$ et
Antognini $^{5}$ ont apporté une contribution majeure à nos connaissances de ce paradigme de l'anesthésie. Rampil a démontré que la suppression d'une réaction de mouvement à un stimulus nociceptif est causée par l'action d'un anesthésique volatil sur un processus rachidien plutôt que sur un processus cérébral. Antognini, quant à lui, a démontré qu'une concentration alvéolaire minimale significativement plus élevée était nécessaire pour prévenir le mouvement lors de l'anesthésie du cerveau d'une chèvre sans anesthésier sa moelle épinière comparativement à une exposition simultanée du cerveau et de la moelle épinière à l'anesthésique volatil. Ces deux découvertes ont clairement indiqué qu'une suppression cruciale de la moelle épinière est nécessaire pour réaliser une anesthésie, mais que la moelle épinière n'est pas responsable de la perte de conscience, un élément essentiel de l'anesthésie générale. Peu après ces travaux, on a observé que les opioïdes réduisaient la concentration alvéolaire minimale (CAM) d'un anesthésique volatil à une valeur égale à la concentration de CAM éveillé. En d'autres mots, si l'on ajoute suffisamment d'opioïdes pendant l'administration d'un anesthésique volatil pour réaliser une anesthésie générale, la concentration d'anesthésique volatil est réduite à celle qui correspond à l'absence de réponse à un stimulus verbal. Cela ressemble à l'utilisation d'une combinaison d'analgésie rachidienne/péridurale et à l'ajout de la quantité nécessaire d'anesthésique volatil pour rendre le patient inconscient. Sur la base de ces données, selon moi l'anesthésie implique premièrement le fait de rendre le patient inconscient par le biais d'un mécanisme situé dans un centre de conscience du cerveau et, deuxièmement, le fait de prévenir un stimulus nociceptif d'atteindre ce centre de conscience. Ces connaissances nous font également réaliser que, si nous souhaitons créer un système automatisé pour surveiller l'anesthésie, nous devons être à même de mesurer les effets de l'anesthésique aussi bien sur la fonction cérébrale que sur la fonction de la moelle épinière. Quel défi!

À la fin des années 1940 et au début des années 1950, Bickford a commencé à évaluer l'électroencéphalogramme (EEG) comme mesure possible de l'effet d'un médicament anesthésique et de sa corrélation avec la perte de conscience. Dès cette époque, un système automatisé d'administration médicamenteuse a commencé à être mis au point pour fournir l'anesthésie. ${ }^{6}$ L'EEG est un schéma d'ondes très complexe que nombre de chercheurs ont tenté de réduire à une valeur dérivée unique (par ex., rebord spectral, fréquence médiane de l'EEG). Avec ces mesures dérivées de l'EEG, une courbe biphasique apparaît pour la plupart des anesthésiques au fur et à mesure qu'ils fournissent une sédation et une perte de conscience plus importantes. L'index bispectral (BIS), un autre indice dérivé de l'EEG, a été conçu spécifiquement comme 
mesure linéaire d'une sédation et d'une perte de conscience croissantes (particulièrement dans la plage de valeurs de 50-100). Ces progrès ont permis de créer un outil de mesure pour au moins une composante de l'état anesthésique, permettant ainsi une surveillance automatisée de cette composante.

Les algorithmes techniques nécessaires à la création d'un système en boucle fermée sont bien connus. Les systèmes biologiques, cependant, ont tendance à être bien plus compliqués. Un système de surveillance automatisé simple que nous connaissons bien est le régulateur de vitesse de nos véhicules. Les défis d'une surveillance d'un système biologique comprennent les changements, variant en fonction du temps, de la relation entre la dose de médicament et la concentration de médicament, outre la variabilité de la sensibilité inter- et intra-individuelle (c.-à-d. la relation changeante entre la concentration du médicament et l'effet). Il n'y a donc pas de relation simple entre administration et résultat obtenu. Plusieurs solutions ont vu le jour pour résoudre ces problèmes, notamment l'utilisation de modèles pharmacocinétiques qui tiennent compte de la relation variant dans le temps entre la dose et la concentration au site effecteur, des contrôles adaptatifs, ou la logique de l'incertain pour tenir compte des différences de sensibilité. ${ }^{7-11}$

Dès lors, des solutions techniques ont été mises au point pour la surveillance automatisée d'un système biologique, et au moins une composante de l'anesthésie peut être placée sous surveillance automatisée. Hemmerling et coll., tout comme plusieurs auteurs avant eux, illustrent l'utilisation d'un système automatisé en boucle fermée pour l'administration de propofol afin de maintenir le BIS dans une marge plus étroite. En fait, le système a donné de meilleurs résultats qu'une surveillance humaine (c.-à-d. manuelle) lorsqu'il s'agissait de maintenir le BIS à une valeur cible ou près de cette valeur. L'algorithme utilisé par les auteurs pour leur système en boucle fermée est quelque peu différent de celui utilisé par d'autres chercheurs, mais il respecte les mêmes principes techniques généraux. Les auteurs ont également montré que le temps de réveil était écourté de trois minutes en moyenne chez les patients auxquels le propofol avait été administré via le système en boucle fermée, ce qui implique que leur système en boucle fermée a eu de meilleurs résultats qu'une administration humaine de propofol aussi bien en termes de surveillance peropératoire de l'hypnose que de devenirs. Est-ce que cela signifie qu'une surveillance automatisée fournit une meilleure anesthésie qu'une surveillance manuelle, constituant ainsi une «réalité actuelle»? Dans un éditorial précédent, publié il y a presque dix ans, et portant sur une évaluation semblable de la surveillance de l'anesthésie par un système automatisé en boucle fermée, j'avais déjà remis en question cette notion, à savoir si le simple fait de démontrer que les systèmes automatisés en boucle fermée sont capables de contrôler précisément une valeur de BIS se traduirait en une démonstration que de tels systèmes sont véritablement meilleurs que des individus pour administrer les médicaments nécessaires à obtenir et maintenir une anesthésie. $^{12}$

Au vu de la nature de l'anesthésie, lorsqu'on évalue la performance d'un système d'anesthésie automatisé en boucle fermée, les attributs observés par les auteurs doivent être évalués de façon critique. Ce que l'étude de Hemmerling et coll. démontre, c'est que l'algorithme de surveillance utilisé dans ce système d'administration de propofol en boucle fermée fonctionne bien (ou pour le moins aussi bien qu'une surveillance humaine) pour maintenir une valeur de BIS prédéterminée/cible et, par conséquent, permet une vitesse de récupération adéquate. Comme cela a été précédemment expliqué, l'anesthésie comporte au moins deux composantes; à ce jour cependant, tous les systèmes automatisés n'ont surveillé que la portion hypnotique de l'anesthésie. L'interaction entre des stimuli nociceptifs, l'analgésie, l'hypnose et l'anesthésie est complexe. ${ }^{13,14} \mathrm{La}$ relation entre l'analgésie et l'hypnose lors de l'administration d'une anesthésie n'est pas linéaire, et la stimulation nociceptive varie constamment tout au long de la chirurgie. Le contrôle de la composante hypnotique exclusivement est utile si l'analgésie est fournie à l'aide d'une anesthésie loco-régionale, de telle manière que seule l'hypnose est nécessaire pour compléter l'état anesthésique. Cependant, si l'on administre des opioïdes pour l'analgésie lors de stimuli nociceptifs intenses, un système en boucle fermée réagirait en augmentant l'agent hypnotique afin de maintenir la valeur de BIS prédéterminée, alors qu'une solution plus adéquate serait de fournir ponctuellement une analgésie plus importante. À ce jour, aucun système en boucle fermée n'est capable de prendre des décisions aussi subtiles. Dans l'étude de Hemmerling et coll., le dosage des opioïdes était à la discrétion de l'anesthésiologiste; par conséquent, le système n'était un système que partiellement en boucle fermée pour l'anesthésie (en d'autres termes, il s'agit véritablement d'un système d'hypnose en boucle fermée plutôt que d'un système d'anesthésie). À cet égard, il est intéressant d'observer que les auteurs ont choisi comme cible une valeur de BIS de 45, soit dans la partie inférieure de la plage de valeurs de BIS recommandées pendant l'anesthésie. Les patients ont reçu un anesthésique «à fort pouvoir hypnotique», minimisant ainsi l'interaction complexe entre l'analgésie de l'hypnose et les stimuli nociceptifs. Par conséquent, tout comme toutes les études publiées à ce jour, celle de Hemmerling et coll. ne démontre pas si les êtres humains, lorsqu'on leur permet de d'optimiser la prise en charge de l'anesthésie et qu'ils 
ne sont pas contraints par une mesure de substitution prédéterminée d'une seule composante de l'anesthésie, sont plus performants que le meilleur système de surveillance en boucle fermée qui soit disponible à l'heure actuelle. De plus, lors des conditions les plus difficiles et variées se manifestant pendant l'anesthésie, un système en boucle fermée peut-il assurer de meilleurs résultats qu'une personne administrant l'anesthésie?

Il est bien plus difficile de répondre à ces questions. Un bon clinicien se servira de la valeur de BIS et d'autres signes d'anesthésie adéquate/inadéquate pour guider l'administration d'agents hypnotiques et analgésiques au patient avec à l'esprit l'intention de fournir une anesthésie avant que le chirurgien ne provoque de stimuli nociceptifs, tout en permettant un prompt retour à l'état conscient et en s'assurant du confort du patient. Dès lors, je tiens à souligner que nous avons établi qu'une hypnose en boucle fermée peut être tout à fait réussie. Néanmoins, il reste à démontrer si l'administration automatisée en boucle fermée d'anesthésiques est véritablement meilleure que ce qu'un professionnel en anesthésie peut réaliser. Pour ce faire, il ne suffit pas de comparer la capacité humaine à atteindre une valeur prédéterminée (c.-à-d. une valeur de BIS) avec celle d'un système en boucle fermée; il convient plutôt de comparer la meilleure anesthésie que chaque professionnel en anesthésie peut administrer. De plus, ces systèmes devraient être mis à l'épreuve dans des conditions physiologiques extrêmes, comme par exemple lors d'épisodes d'hypotension/hypertension, etc. Alors seulement pourrons-nous déterminer si une surveillance automatisée de l'anesthésie constitue une innovation futuriste ou une réalité actuelle. Je suis optimiste quant au fait qu'une surveillance en boucle fermée de l'anesthésie s'avérera en fin de compte supérieure et deviendra une partie intégrante de la prestation de routine d'anesthésie.

Competing interests None declared.

\section{References}

1. Snow J. On the Inhalation of the Vapour of Ether in Surgical Operations. London: John Churchill; 1847.

2. Hemmerling TM, Charabati S, Zaouter C, Minardi C, Mathieu $P A$. A randomized controlled trial demonstrates that a novel closed-loop propofol system performs better hypnosis control than manual administration. Can J Anesth 2010; 57. doi:10.1007/ s12630-010-9335-z.

3. Rampil IJ. Anesthetic potency is not altered after hypothermic spinal cord transection in rats. Anesthesiology 1994; 80: 606-10.

4. Rampil IJ, Mason P, Singh H. Anesthetic potency (MAC) is independent of forebrain structures in the rat. Anesthesiology 1993; 78: 707-12.

5. Antognini JF, Schwartz, K. Exaggerated anesthetic requirements in the preferentially anesthetized brain. Anesthesiology 1993; 79: 1244-9.

6. Bickford RG. Automatic electroencephalographic control of general anesthesia. Electroencephalogr Clin Neurophysiol 1950; 2: $93-6$.

7. Schwilden H, Schuttler J, Stoeckel H. Closed-loop feedback control of methohexital anesthesia by quantitative EEG analysis in humans. Anesthesiology 1987; 67: 341-7.

8. Struys MM, De Smet T, Versichelen LF, Van De Velde S, Van den Broecke R, Mortier EP. Comparison of closed-loop controlled administration of propofol using bispectral index as the controlled variable versus "standard practice" controlled administration. Anesthesiology 2001; 95: 6-17.

9. Kenny GN, Mantzaridis $H$. Closed-loop control of propofol anaesthesia. Br J Anaesth 1999; 83: 223-8.

10. Sakai T, Matsuki A, White PF, Giesecke AH. Use of an EEGbispectral delivery system for administering propofol. Acta Anaesthesiol Scand 2000; 44: 1007-10.

11. Absalom AR, Sutcliffe N, Kenny GN. Closed-loop control of anesthesia using bispectral index: performance assessment in patients undergoing major orthopedic surgery under combined general and regional anesthesia. Anesthesiology 2002; 96: 67-73.

12. Glass PS, Rampil IJ. Automated anesthesia: fact or fantasy? Anesthesiology 2001; 95: 1-2.

13. McEwan AI, Smith C, Dyar O, Goodman D, Smith LR, Glass PS. Isoflurane minimum alveolar concentration reduction by fentanyl. Anesthesiology 1993; 78: 864-9.

14. Minto CF, Schnider TW, Short TG, Gregg KM, Gentilini A, Shafer SL. Response surface model for anesthetic drug interactions. Anesthesiology 2000; 92: 1603-16. 